\title{
HIGHER ORDER ABSOLUTE DIFFERENTIATION WITH RESPECT TO GENERALIZED CONNECTIONS
}

\author{
IVAN KOLÁ $\check{R}$ \\ Institute of Mathematics, Czechoslovak Academy of Sciences, \\ Brno Branch, Brno, Czechoslovakia
}

Given an arbitrary fibered manifold $\pi: Y \rightarrow X$, a connection on $Y$ (or a generalized connection) means any section $\Gamma: Y \rightarrow J^{1} Y$ (= the first jet prolongation of $Y$ ), [3], [5]. Analogously, a non-holonomic $r$ th order (generalized) connection on $Y$ is a section $Y \rightarrow \tilde{J}^{r} Y$, where the $r$ th non-holonomic prolongation $\tilde{J}^{r} Y$ of $Y$ is defined by the iteration $\tilde{J}^{1} Y=J^{1} Y, \tilde{J}^{r} Y=J^{1}\left(\tilde{J}^{r-1} Y\right)$. In fact, the first order absolute differentiation with respect to a generalized connection $\Gamma$ is of the same form as in the classical case of a principal connection. On the other hand, the absolute differentiation of higher order requires quite new procedures. We first explain how such an operation can be defined in terms of the successive vertical prolongations of $\Gamma$. However, this approach has somewhat "static" character and cannot be applied to higher order connections. That is why we develop a "kinematic" approach based on the idea of a parametrized jet. (It seems that the latter concept can be useful in some other higher order theories as well.) Then we deduce that the iterated absolute differentiation with respect to the vertical prolongations corresponds to the construction of the product of connections, [8]. In particular, the use of the successive vertical prolongations of a first order connection $\Gamma$ is equivalent to the use of the prolongations of $\Gamma$ in the sense of Ehresmann, [1], [2].

\section{Vertical prolongations}

Consider a connection $\Gamma: Y \rightarrow J^{1} Y$. In local fiber coordinates $x^{i}, y^{p}$ on $Y$, the equations of $\Gamma$ are

$$
d y^{p}=F_{i}^{p}(x, y) d x^{i}
$$

with arbitrary smooth (i.e., infinitely differentiable) functions $F_{i}^{p}$. Given a vector field $\xi$ on $X$, denote by $\Gamma \xi$ the $\Gamma$-lift of $\xi$. Using flows, we prolong $\Gamma \xi$ into a vector field $V(\Gamma \xi)$ on the vertical tangent bundle $V Y$ of $Y$, [4]. The mapping $\xi \mapsto V(\Gamma \xi)$ 
determines lifting with respect to a unique connection $V I$ on $V Y \rightarrow X$ with the following equations

$$
d y^{p}=F_{i}^{p}(x, y) d x^{i}, \quad d \eta^{p}=\frac{\partial F_{i}^{p}(x, y)}{\partial y^{q}} \eta^{q} d x^{i},
$$

where $\eta^{p}=d y^{p}$ are the induced coordinates on $V Y$. By iteration, we obtain a connection $V^{r} \Gamma=V\left(V^{r-1} \Gamma\right)$ on the $r$ th vertical tangent bundle $V^{r} Y=V\left(V^{r-1} Y\right)$.

The connection map $\omega_{\Gamma}: T Y \rightarrow V Y$ assigns to every vector of $T_{y} Y$ its projection into $V_{y} Y$ in the direction $\Gamma(y), y \in Y$. The equations of $\omega_{\Gamma}$ are

$$
\eta^{p}=d y^{p}-F_{i}^{p}(x, y) d x^{i} .
$$

Given a manifold $M$ and a smooth map $f: M \rightarrow Y$, the composition $\nabla_{\Gamma} f$ of the tangent map $T f: T M \rightarrow T Y$ and $\omega_{\Gamma}$ is said to be the absolute differential of $f$ with respect to $\Gamma, \nabla_{\Gamma} f:=\omega_{\Gamma} \circ T f: T M \rightarrow V Y$. Denoting by

$$
J^{1}(M, Y, \pi)=\bigcup_{x \in X} J^{1}\left(M, Y_{x}\right)
$$

the space of all 1-jets of $M$ into the individual fibers of $Y$, the absolute differential of $f$ can also be interpreted as a mapping $\nabla_{\Gamma} f: M \rightarrow J^{1}(M, Y, \pi)$.

Taking into account $V \Gamma$ and $\nabla_{\Gamma} f: T M \rightarrow V Y$, we can construct the absolute differential $\nabla_{\Gamma}^{2} f:=\nabla_{V \Gamma}\left(\nabla_{\Gamma} f\right): T T M \rightarrow V V Y$ called the second absolute differential of $f$ with respect to $\Gamma$. By iteration, we obtain the $r$ th absolute differential of $f$ with respect to $\Gamma$

$$
\nabla_{T}^{r} f:=\nabla_{V^{r-1}}\left(\nabla_{\Gamma}^{r-1} f\right): T^{(r)} M \rightarrow V^{r} Y,
$$

where $T^{(r)} M=\underbrace{T \ldots T M}_{r \text {-times }}$. We recall that every non-holonomic $r$-jet $A \in \tilde{J}_{p}(M, N)_{q}$ determines a map $A_{*}: T_{p}^{(r)} M \rightarrow T_{q}^{(r)} N$ by the following induction, cf. [7]. In the first step, the identification $J_{p}^{1}(M, N)_{q}=\operatorname{Hom}\left(T_{p} M, T_{q} N\right)$ is trivial. For $r>1$, we have $A=j_{p}^{1} f$, where $f$ is a section $M \rightarrow \tilde{J}^{r-1}(M, N)$. By the induction hypothesis, $f$ determines a map $f_{*}: T^{(r-1)} M \rightarrow T^{(r-1)} N$ and we set

$$
A_{*}=T_{p}\left(f_{*}\right): T_{p}^{(r)} M \rightarrow T_{q}^{(r)} N .
$$

Analyzing (4), we prove

Proposition 1. For every $p \in M$, there exists a unique semiholonomic r-jet $A \in \bar{J}_{p}^{r}\left(M, Y_{x}\right), x=\pi f(p)$, satisfying

$$
A_{*}=\nabla_{I}^{r} f(p): T_{p}^{(r)} M \rightarrow V_{f(p)}^{r} Y=T_{f(p)}^{r}\left(Y_{x}\right) .
$$

(We remark that Proposition 1 follows immediately from formula (26) below.) Hence we can also write

$$
\nabla_{\Gamma}^{r} f: M \rightarrow \bar{J}^{r}(M, Y, \pi):=\bigcup_{x \in X} \bar{J}^{r}\left(M, Y_{x}\right) .
$$

In particular, if $\sigma$ is a section of $Y$ with equations $y^{p}=\sigma^{p}(x)$, then we deduce by (2)-(4) that the coordinates of $\nabla_{\Gamma}^{2} f$ are $\partial \sigma^{p} / \partial x^{i}-F_{i}^{p}(x, \sigma)$ and

$$
\frac{\partial^{2} \sigma^{p}}{\partial x^{i} \partial x^{j}}-\frac{\partial F_{i}^{p}}{\partial x^{j}}-\frac{\partial F_{i}^{p}}{\partial y^{q}} \frac{\partial \sigma^{q}}{\partial x^{j}}-\frac{\partial F_{j}^{p}}{\partial y^{q}} \frac{\partial \sigma^{q}}{\partial x^{i}}+\frac{\partial F_{j}^{p}}{\partial y^{q}} F_{i}^{q} .
$$


We recall that the curvature of $\Gamma$ is a section $\Omega_{\Gamma}: Y \rightarrow V Y \otimes \bigwedge^{2} T^{*} X$ with the following coordinate expression, [3],

$$
\eta^{p}=\left(\partial_{j} F_{i}^{p}+F_{j}^{q} \partial_{q} F_{i}^{p}\right) d x^{i} \wedge d x^{j}
$$

Applying alternation to (6), we obtain

Proposition 2. For every local section $\sigma$ of $Y, \sigma(x)=y$, we have

$$
\Delta\left(\nabla_{\Gamma}^{2} \sigma(x)\right)=-\Omega_{\Gamma}(y),
$$

where $\Delta$ means the difference tensor, [2].

\section{Parametrized jets}

Let $M, N, P$ be manifolds and $C^{\infty}(N, P)$ the space of all smooth maps of $N$ into $P$. (Hence $C^{\infty}(N, P)$ is not a manifold in the classical sense.) A map $f: M \rightarrow C^{\infty}(N, P)$ will be called smooth if the induced map

$$
\bar{f}: M \times N \rightarrow P, \quad \bar{f}(p, q)=f(p)(q), \quad p \in M, q \in N,
$$

is smooth; the set of all such maps will be denoted by $C^{\infty}\left(M, C^{\infty}(N, P)\right)$. We shall say that two smooth maps $f, g: M \rightarrow C^{\infty}(N, P)$ are r-equivalent at $p \in M$, if

$$
j_{p}^{r} \bar{f}(-, q)=j_{p}^{r} \bar{g}(-, q) \in J_{p}^{r}(M, P) \quad \text { for all } q \in N .
$$

The equivalence class $A=j_{p}^{r} f$ will be called $r$-jet of $M$ into $C^{\infty}(N, P), p=\alpha A$ or $f=\beta A$ is the source or target of $A$, respectively. Similarly to the classical case, we write $J^{r}\left(M, C^{\infty}(N, P)\right), J_{p}^{r}\left(M, C^{\infty}(N, P)\right)_{f}$, etc.

Every $j_{p}^{r} f \in J_{p}^{r}\left(M, C^{\infty}(N, P)\right)$ determines a map $j_{p}^{r} \bar{f}(-, q): N \rightarrow J_{p}^{r}(M, P)$. Conversely, given $F: N \rightarrow J_{p}^{r}(M, P)$, one can prove that there is a neighbourhood $p \in U \subset M$ and $f: U \rightarrow C^{\infty}(N, P)$ such that $F(q)=j_{p}^{r} \bar{f}(-, q)$. Thus, we have an identification

$$
J_{p}^{r}\left(M, C^{\infty}(N, P)\right)=C^{\infty}\left(N, J_{p}^{r}(M, P)\right) .
$$

That is why the jets of this type will be said to be parametrized jets. Obviously, any element of $J_{p}^{r}\left(M, C^{\infty}(N, P)\right)_{f}$ is a mapping $F: N \rightarrow J_{p}^{r}(M, P)$ satisfying $\beta F=f$.

EXAMPLE 1. The tangent space $T_{p} M$ of a manifold $M$ can be defined as $T_{p}(M)$ $=J_{0}^{1}(R, M)_{p}$. If we introduce similarly $T_{f} C^{\infty}(N, P)=J_{0}^{1}\left(R, C^{\infty}(N, P)\right)_{f}$, we obtain the standard interpretation of the elements of $T_{f} C^{\infty}(N, P)$ as mappings $F: N \rightarrow T P$ over $f$, which are called vector fields along $f$. In this case, identification (9) gives $T\left(C^{\infty}(N, P)\right)=C^{\infty}(N, T P)$.

If $Q$ is another manifold and $B=j_{t}^{r} g \in J_{t}^{r}(Q, M)_{p}$ an ordinary jet, we have the composition

$$
A \circ B:=j_{t}^{r}(f \circ g) \in J_{t}^{r}\left(Q, C^{\infty}(N, P)\right),
$$

$A=j_{p}^{r} f \in J_{p}^{r}\left(M, C^{\infty}(N, P)\right)$. Further, any smooth map $F: P \rightarrow Q$ transforms $j_{p}^{r} f$ into

$$
F \circ\left(j_{p}^{r} f(u)\right)=j_{p}^{r}(F \circ f(u)) \in J^{r}\left(M, C^{\infty}(N, Q)\right), \quad u \in M .
$$


On the other hand, any $F \in C^{\infty}(Q, N)$ transforms $j_{p}^{r} f$ into

$$
\left(j_{p}^{r} f(u)\right) \circ F=j_{p}^{r}(f(u) \circ F) \in J^{r}\left(M, C^{\infty}(Q, P)\right) .
$$

Consider a fibered manifold $\pi: Y \rightarrow X$ and the space

$$
C^{\infty}(N, Y, \pi):=\bigcup_{x \in X} C^{\infty}\left(N, Y_{x}\right) \subset C^{\infty}(N, Y)
$$

of all smooth maps of $N$ into the individual fibers of $Y$. We have an induced map $b: C^{\infty}(N, Y, \pi) \rightarrow X$. Obviously, we have

$$
J^{r}\left(M, C^{\infty}(N, P)\right)=C^{\infty}\left(N, J^{r}(M, P), \alpha\right),
$$

$\alpha: J^{r}(M, P) \rightarrow M$. A mapping $f: Q \rightarrow J^{r}\left(M, C^{\infty}(N, P)\right)$ will be called smooth if it is smooth under identification (13).

The non-holonomic parametrized jets can be introduced by the following induction. For $r=1$, we have the above holonomic case. Given a smooth section

$$
f: M \rightarrow \tilde{J}^{r-1}\left(M, C^{\infty}(N, P)\right),
$$

$j_{p}^{1} f$ will be called a non-holonomic r-jet of $M$ into $C^{\infty}(N, P)$. Analogously to (13), we have

$$
\tilde{J}^{r}\left(M, C^{\infty}(N, P)\right)=C^{\infty}\left(N, \tilde{J}^{r}(M, P), \alpha\right) .
$$

Formulae (10)-(12) are naturally extended to non-holonomic jets. The semi-holonomic parametrized jets are introduced in standard way.

If $A \in \tilde{J}_{p}^{r}\left(M, C^{\infty}(N, Y, \pi)\right)$, then $b A \in \tilde{J}_{p}^{r}(M, X)$ is an ordinary $r$-jet. Denoting by

$$
\tilde{\pi}_{M}^{r}: \tilde{J}^{r}(M, Y) \rightarrow \tilde{J}^{r}(M, X)
$$

the map derived from $\pi$, we easily find an identification

$$
\tilde{J}_{p}^{r}\left(M, C^{\infty}(N, Y, \pi)\right)=C^{\infty}\left(N, \tilde{J}_{p}^{r}(M, Y), \tilde{\pi}_{M}^{r}\right) .
$$

In other words, the elements of $\tilde{J}_{p}^{r}\left(M, C^{\infty}(N, Y, \pi)\right)$ are maps of $N$ into $\tilde{J}_{p}^{r}(M, Y)$ over the same element of $\tilde{J}_{p}^{r}(M, X)$. We shall use the symbol

$$
\tilde{J}_{p}^{r}\left(M, C^{\infty}(N, Y, \pi)\right)_{f, B} \subset \tilde{J}_{p}^{r}\left(M, C^{\infty}(N, Y, \pi)\right)_{f}, \quad B \in \tilde{J}_{p}^{r}(M, X),
$$

for the subspace of all jets over $B$.

EXAMPLE 2. Take $N=Y_{x}, f=e_{x}=\mathrm{id}_{Y_{x}}, M=R, p=0$ and put

$$
L_{x} Y=J_{0}^{1}\left(R, C^{\infty}\left(Y_{x}, Y, \pi\right)\right)_{e_{x}} .
$$

Every element of $L_{x} Y$ is a vector field along $Y_{x}$ over the same vector of $T_{x} X$, i.e., a projectable vector field along $Y_{x}$.

\section{Fiber jets}

We shall say that two maps $f, g: Y \rightarrow N$ have $r$-th order fiber contact at $x \in X$ if $j_{y}^{r} f=j_{y}^{r} g$ for all $y \in Y_{x}$. Such an equivalence class $j_{x}^{r} f$ will be called a fiber $r$-jet of $Y$ into $N$. Using local coordinates, we easily find that any two of the following three conditions are equivalent: 
(i) $j_{x}^{r} f=j_{x}^{r} g$,

(ii) $j_{x}^{r}(f \circ \sigma)=j_{x}^{r}(g \circ \sigma) \in J_{x}^{r}(X, N)$ for all local sections $\sigma$ of $Y$,

(iii) for any local trivialization of $Y, j_{x}^{r} f(-, y)=j_{x}^{r} g(-, y) \in J_{x}^{r}(X, N)$ for all $y \in Y_{x}$.

If $v^{\lambda}$ are some local coordinates on $N$, then a fiber jet of $Y$ into $N$ is determined by smooth functions $f^{\lambda}(y), f_{i}^{\lambda}(y), \ldots, f_{i_{1} \ldots i_{r}}^{\lambda}(y)$ defined on the corresponding fiber of $Y$ and symmetric in all subscripts.

Given another fibered manifold $\varrho: W \rightarrow Z$, we denote by $\underline{J}^{r}(Y, W)$ the set of all fiber $r$-jets of fibered manifold morphisms of $Y$ into $W$. If $U \rightarrow V$ is a third fibered manifold and $f: Y \rightarrow W$ or $g: W \rightarrow U$ a morphism over $f_{0}: X \rightarrow Z$ or $g_{0}: Z \rightarrow V$, respectively, then we define

$$
\left(j_{z}^{r} g\right) \circ\left(j_{x}^{r} f\right)=j_{x}^{r}(g \circ f), \quad z=f_{0}(x) .
$$

Hence the fiber $r$-jets of fibered manifold morphisms form a category $\underline{J}^{r}$ with a canonical functor into the category $J^{r}$ of ordinary $r$-jets.

Let $C^{\infty}(\pi, Y, N):=\bigcup_{x \in X} C^{\infty}\left(Y_{x}, N\right)$ be the space of all smooth maps of the individual fibers of $Y$ into $N$ and $a: C^{\infty}(\pi, Y, N) \rightarrow X$ the natural projection. A map $f: M \rightarrow C^{\infty}(\pi, Y, N)$ will be called smooth if

(a) af: $M \rightarrow X$ is smooth,

(b) the derived map $\bar{f}:(a f)^{-1} Y \rightarrow N, \bar{f}(p, q)=f(p)(q)$ is smooth, provided $(a f)^{-1} Y$ means the induced fibered manifold over $M$. In particular, a section $\sigma: X$ $\rightarrow C^{\infty}(\pi, Y, N)$ is smooth if the derived map $\bar{\sigma}: Y \rightarrow N, \vec{\sigma}(y)=\sigma(\pi y)(y)$ is smooth.

EXAMPLE 3. Set $L Y=\bigcup_{x \in X} L_{x} Y$. A smooth section $\sigma: X \rightarrow L Y$ corresponds to a projectable vector field on $Y$. The bracket of such fields defines a bracket operation on the sections of $L Y$, what endows $L Y$ with a Lie algebroid structure in the sense of [6]. We remark that $L Y$ is the Lie algebroid of the groupoid $G Y$ defined in $\S 4$ below.

Let $\mu: S \rightarrow X$ be a fibered manifold and $F: S \rightarrow Y$ a base-preserving morphism. Then we have an induced map

$$
F^{*}: C^{\infty}\left(M, C^{\infty}(\pi, Y, N)\right) \rightarrow C^{\infty}\left(M, C^{\infty}(\mu, S, N)\right), \quad\left(F^{*} f\right)(p)=f(p) \circ\left(F \mid S_{f(p)}\right) .
$$

In particular, any section $\sigma: X \rightarrow Y$ determines a map $\sigma^{*} f: M \rightarrow N$.

Two smooth maps $f, g: M \rightarrow C^{\infty}(\pi, Y, N)$ will be called requivalent at $p \in M$ if $j_{p}^{r}\left(\sigma^{*} f\right)=j_{p}^{r}\left(\sigma^{*} g\right) \in J_{p}^{r}(M, N)$ for every local section $\sigma$ of $Y$. If $a f=a g=: h$, this condition is equivalent to the fact that the derived maps $\bar{f}, \bar{g}: h^{-1} Y \rightarrow N$ have $r$ th order fiber contact at $p$. In this situation, we shall also use the classical notation $j_{p}^{r} f, J_{p}^{r}\left(M, C^{\infty}(\pi, Y, N)\right)$, etc. In local coordinates $u^{\alpha}$ on $M$, an element of $J^{r}\left(M, C^{\infty}(\pi, Y, N)\right)$ is determined by smooth functions $f^{\lambda}(y), f_{\alpha}^{\lambda}(y), \ldots, f_{\alpha_{1} \ldots \alpha_{r}}^{\lambda}(y)$ on the corresponding fiber of $Y$.

To introduce non-holonomic fiber jets, we first present the construction of ordinary non-holonomic jets in a convenient form. First of all, any $A \in \tilde{J}_{p}^{2}(M, N)$ 
is of the form $A=j_{p}^{1} F$, where $F$ is a section $M \rightarrow J^{1}(M, N)$. Every $F\left(p_{2}\right), p_{2} \in M$, is defined by $F\left(p_{2}\right)=j_{p_{2}}^{1} f\left(-, p_{2}\right)$. Hence we have a smooth mapping $f: M \times M \rightarrow N$ and we can write $A=\tilde{j}_{p}^{2} f:=j_{p}^{1}\left(j_{p_{2}}^{1} f\left(-, p_{2}\right)\right) \in \tilde{J}_{p}^{2}(M, N)$. For arbitrary $r$, we take similarly a map $f: \dot{\times} M \rightarrow N$ and construct $\tilde{j}_{p}^{r} f=j_{p}^{1} j_{p_{2}}^{1} \ldots j_{p_{r}}^{1} f \in \tilde{J}_{p}^{r}(M, N)$.

The space $\tilde{J}^{r}\left(M, C^{\infty}(\pi, Y, N)\right)$ is defined by the following induction. For $r=1$, we have the above holonomic case. Assume by induction that we have defined $\tilde{j}_{p}^{r-1} f \in \tilde{J}^{r-1}\left(M, C^{\infty}(\pi, Y, N)\right)$ for any smooth map $f: \stackrel{r-1}{X} M \rightarrow C^{\infty}(\pi, Y, N)$. A section

$$
\varphi: M \rightarrow \tilde{J}^{r-1}\left(M, C^{\infty}(\pi, Y, N)\right)
$$

will be called smooth if there is a smooth map

such that

$$
f: M \times \stackrel{r-1}{\chi}^{X} M \rightarrow C^{\infty}(\pi, Y, N)
$$

for all $u \in M$. Let

$$
\varphi(u)=\tilde{j}_{u}^{r-1} f(u,-)
$$

$$
\psi: M \rightarrow \tilde{J}^{r-1}\left(M, C^{\infty}(\pi, Y, N)\right), \quad \psi=\tilde{j}_{u}^{r-1} g(u,-)
$$

be another smooth section. Then we define

$$
j_{p}^{1} \varphi=j_{p}^{1} \psi \in \tilde{J^{r}}\left(M, C^{\infty}(\pi, Y, N)\right)
$$

by requiring

$$
\tilde{j}_{p}^{r} \sigma^{*} f=\tilde{j}_{p}^{r} \sigma^{*} g \in \tilde{J}_{p}^{r}(M, N)
$$

for every local section $\sigma$ of $Y$.

If $\varrho: W \rightarrow Z$ is another fibered manifold, then

$$
C^{\infty}(\pi, Y, W, \varrho) \subset C^{\infty}(\pi, Y, W)
$$

means the subspace of all smooth maps of one fiber of $Y$ into one fiber of $W$. Any $C \in \tilde{J}_{p}^{r}\left(M, C^{\infty}(\pi, Y, W, \varrho)\right)$ determines $a C \in \tilde{J}_{p}^{r}(M, X)$ and $b C \in \tilde{J}_{p}^{r}(M, Z)$. Given $A \in \tilde{J}_{p}^{r}(M, X)$ and $B \in \tilde{J}_{p}^{r}(M, Z)$, we denote by $\tilde{J}_{p, A}^{r}\left(M, C^{\infty}(\pi, Y, W, \varrho)\right)_{f, B}$ $\subset \tilde{J}_{p}^{r}\left(M, C^{\infty}(\pi, Y, W, \varrho)\right)_{f}$ the subspace of all jets over $A$ and $B$. Any element $A \in \tilde{J}_{x}^{r}\left(X, C^{\infty}(\pi, Y, W, \varrho)\right)$ satisfying $a A=j_{x}^{r} \mathrm{id}_{x}=: E_{x}^{r}$ will be called non-holonomic fiber r-jet of $Y$ into $W$ and $\tilde{J}^{r}(Y, W)$ will denote the space of all such jets. If $B$ $\in \underline{\tilde{J}}_{z}^{r}(W, U), z=b \beta A$, we define $B \circ A \in \underline{\tilde{J}}^{r}(Y, U)$ by iterating (17). Thus we obtain a category $\tilde{\tilde{J}}^{r}$ of non-holonomic fiber $r$-jets.

Consider further $A \in \tilde{J}_{p}^{r}(M, Y)$ and $B \in \tilde{J}_{p}^{r}\left(M, C^{\infty}(\pi, Y, N)\right)$ satisfying $\pi A$ $=a B \in \tilde{J}_{p}^{r}(M, X)$. We may write $\pi A=a B=\tilde{j}_{p}^{r} h, A=\tilde{j}_{p}^{r} f, B=\tilde{j}_{p}^{r} g$, where $f$ or $g$ is a map of $\stackrel{r}{X} M$ into $Y$ or $C^{\infty}(\pi, Y, N)$ over the same map $h: \stackrel{r}{X} M \rightarrow X$. Then

$$
(g \cdot f)\left(p_{1}, \ldots, p_{r}\right)=g\left(p_{1}, \ldots, p_{r}\right)\left(f\left(p_{1}, \ldots, p_{r}\right)\right)
$$

is a mapping of $\stackrel{r}{X} M$ into $N$ and we define

$$
B \cdot A=\tilde{j}_{p}^{r}(g \cdot f) \in \tilde{J}_{p}^{r}(M, N) .
$$


Assume that $Y$ and $W$ have diffeomorphic fibers and denote by

$$
I^{\infty}(\pi, Y, W, \varrho) \subset C^{\infty}(\pi, Y, W, \varrho)
$$

the subspace of all smooth diffeomorphisms. For every

$$
A=j_{p}^{r} f \in J_{p}^{r}\left(M, I^{\infty}(\pi, Y, W, \varrho)\right),
$$

we introduce its inverse

$$
A^{-1}:=j_{p}^{r}\left(f^{-1}\right) \in J_{p}^{r}\left(M, I^{\infty}(\varrho, W, Y, \pi)\right),
$$

where $f^{-1}$ is the inverse diffeomorphism of $f$. For non-holonomic $r$-jets of $M$ into $I^{\infty}(\pi, Y, W, \varrho)$, such an inversion is defined by standard induction based on (19).

\section{Elements of connection}

Consider the groupoid $G Y:=I^{\infty}(\pi, Y, Y, \pi)$ of all diffeomorphisms between the individual fibers of $Y$. Using an idea by Ehresmann, [1], we define the space of all non-holonomic $r$ th order elements of connection on $Y$ at $x$ by

$$
\tilde{Q}_{x}^{r} Y:=\tilde{J}_{x, 0_{x}^{r}}^{r}(X, G Y)_{e_{x}, E_{x}^{r}},
$$

where $0_{x}^{r}$ means the $r$-jet at $x$ of the constant map $t \mapsto x, t \in X$. Since any $A \in \tilde{Q}_{x}^{r} Y$ satisfies $a A=0_{x}^{r}$, we can also express $\tilde{Q}_{x}^{r} Y$ as

$$
\tilde{Q}_{x}^{r} Y=\tilde{J}_{x}^{r}\left(X, C^{\infty}\left(Y_{x}, Y, \pi\right)\right)_{e_{x}, E_{x}^{r}} .
$$

By (15), every $C \in \tilde{Q}_{x}^{r} Y$ is identified with a section $\Gamma: Y_{x} \rightarrow \tilde{J}^{r} Y$. Hence any smooth section

$$
C: X \rightarrow \tilde{Q}^{r} Y:=\bigcup_{x \in X} \tilde{Q}_{x}^{r} Y
$$

is identified with a section $\Gamma: Y \rightarrow \tilde{J}^{r} Y$, i.e., $C$ is a non-holonomic $r$ th order connection on $Y$.

The absolute differential $\nabla_{A} B$ of $B \in \tilde{J}_{p}^{r}(M, Y)$ with respect to an element of connection $A \in \tilde{Q}_{x}^{r} Y, \quad x=\pi \beta B$, is defined as follows. We have $A^{-1}$ $\in \tilde{J}_{x, E_{x}^{r}}^{r}(X, G Y)_{e_{x}, 0_{x}^{r}}$ and $\pi B \in \tilde{J}_{p}^{r}(M, X)_{x}$, so that we can construct $\left(A^{-1} \circ(\pi B)\right) \cdot B$ $\in \tilde{J}_{p}^{r}(M, Y)$ by $(10)$ and (18). Since $b A^{-1}=0_{x}^{r}$, the latter jet belongs to $\tilde{J}_{p}^{r}\left(M, Y_{x}\right)$. Then we set

$$
\nabla_{A} B:=\left(A^{-1} \circ(\pi B)\right) \cdot B \in \tilde{J}_{p}^{r}\left(M, Y_{x}\right) .
$$

In particular, if $B \in \tilde{J}^{r} Y$, then (22) is simplified to $\nabla_{A} B=A^{-1} \cdot B$. Given a map $f: M \rightarrow Y$ and a connection $I: Y \rightarrow \tilde{J^{r}} Y$, we use the corresponding section $C: X \rightarrow \tilde{Q}^{r} Y$ to define

$$
\nabla_{\Gamma} f(p)=\nabla_{C(x)} j_{p}^{r} f \in \tilde{J}_{p}^{r}\left(M, Y_{x}\right), \quad x=\pi f(p) .
$$

The map $\nabla_{\Gamma} f: M \rightarrow \tilde{J}(M, Y, \pi)$ is called the absolute differential of $f$ with respect to $\Gamma$. 


\section{Product of connections}

Consider an $r$ th order connection $C: X \rightarrow \tilde{Q}^{r} Y$ and a first order connection $D: X \rightarrow Q^{1} Y$. Let

Since

$$
D(x)=j_{x}^{1} \varphi(t), \quad \varphi(t): Y_{x} \rightarrow Y_{t} .
$$

we have

$$
C(t) \in \tilde{J}_{t}^{r}\left(X, C^{\infty}\left(Y_{t}, Y, \pi\right)\right)_{e_{t}, E_{t}^{r}},
$$

by (12). Then

$$
C(t) \circ \varphi(t) \in \tilde{J_{t}^{r}}\left(X, C^{\infty}\left(Y_{x}, Y, \pi\right)\right)
$$

$$
j_{x}^{1}(C(t) \circ \varphi(t)) \in \tilde{J}_{x}^{r+1}\left(X, C^{\infty}\left(Y_{x}, Y, \pi\right)\right)_{e_{x}, E_{x}^{r+1}}=\tilde{Q}_{x}^{r+1} Y .
$$

Hence we obtain a non-holonomic $(r+1)$ st order connection $C * D: X \rightarrow \tilde{Q}^{r+1} Y$. (For principal connection, this operation was introduced by Virsik, [8].) Taking into account the corresponding sections $\Gamma: Y \rightarrow \tilde{J}^{r} Y$ and $\Delta: Y \rightarrow J^{1} Y$, we deduce by (24)

Proposition 3. The section $\Gamma * \Delta: Y \rightarrow \tilde{J}^{r+1} Y$ corresponding to $C * D: X \rightarrow \tilde{Q^{r+1}} Y$ is $\Gamma * \Delta=J^{1} \Gamma \circ \Delta$, where $J^{1} \Gamma: J^{1} Y \rightarrow \tilde{J}^{r+1} Y$ is the jet prolongation of $\Gamma: Y \rightarrow \tilde{J}^{r} Y$.

In particular, if $\Gamma$ is a first order connection on $Y$, then $\Gamma^{(r-1)}=\underbrace{\Gamma * \Gamma_{*} \ldots * \Gamma}_{r \text {-times }}$ is the $(r-1)$ st prolongation of $\Gamma$ in the sense of Ehresmann, [1]. The values of $\Gamma^{(r-1)}$ lie in the $r$ th semi-holonomic prolongation of $Y$, [2].

Given $B \in \tilde{J}_{p}^{r+1}(M, Y)$, we determine a construction of the absolute differential $\nabla_{C * D(x)} B, x=\pi \beta B$. We have $B=j_{p}^{1} F$, where $F$ is a section $M \rightarrow \tilde{J}^{r}(M, Y)$. Using $\Gamma: Y \rightarrow \tilde{J}^{r} Y$, we construct $\nabla_{\Gamma} F: M \rightarrow \tilde{J}^{r}(M, Y, \pi)$. By (8), $\nabla_{T} F$ can be interpreted as a map $T^{(r)} M \rightarrow V^{r} Y$. Taking into account the $r$ th vertical prolongation $V^{r} \Delta$ of $\Delta$, we get

$$
\nabla_{V^{r} \Delta}\left(\nabla_{T} F\right)(p): T_{p}^{(r+1)} M \rightarrow V^{r+1} Y,
$$

which corresponds to an element of $\tilde{J}_{p}^{r+1}\left(M, Y_{x}\right)$. By the definition of $V^{r} \Delta$, we deduce

Proposition 4. We have

$$
\nabla_{C * D(x)} B=\nabla_{V^{r} \Gamma}\left(\nabla_{\Gamma} F\right)(p) .
$$

In particular, if we have $r$ first order connections $\Gamma_{1}, \Gamma_{2}, \ldots, \Gamma_{r}$ and consider their product $\Gamma_{1} * \Gamma_{2} * \ldots * \Gamma_{r}$, Proposition 4 implies

Proposition 5. For any $f: M \rightarrow Y$, we have

$$
\nabla_{\Gamma_{1} * \Gamma_{2} * \ldots * \Gamma_{r}} f=\nabla_{V^{r-1} \Gamma_{r}} \ldots \nabla_{V \Gamma_{2}} \nabla_{\Gamma_{1}} f .
$$

If $\Gamma_{1}=\Gamma_{2}=\ldots=\Gamma_{r}=\Gamma$, then the right-hand side of (25) is $\nabla_{I}^{r} f$ in the sense of $\S 1$. Hence we have

$$
\nabla_{\Gamma}^{r} f=\nabla_{\Gamma^{(r-1)} f}
$$

This formula shows that the $r$ th order absolute differentiation with respect to a first order connection $\Gamma$ on $Y$ in the sense of Section 1 coincides with the absolute differentiation with respect to $\Gamma^{(r-1)}$. 


\section{References}

[1] C. E h r es m a n n, Sur les connexions d'ordre supérieur, Atti del $\mathrm{V}^{\circ}$ Congresso dell'Unione Matematica Italiana, 1955, Roma Cremonese 344-346.

[2] I. Ko lá r̆, On the torsion of spaces with connection, Czechoslovak Math. J. 21 (1971), 124-136.

[3] -, On generalized connections, Beiträge zur Algebra und Geometrie 11 (1981), 29-34.

[4] —, Connections in 2-fibered manifolds, Arch. Math. (Brno) 17 (1981), 23-30.

[5] P. Li be r m a n n, Parallélismes, J. Differential Geometry 8 (1973), 511-539.

[6] J. Pradines, Théorie de Lie pour les groupoides différentiables. Calcul différentiel dans la catégorie des groupoides infinitésimaux, C. R. Acad. Sci. Paris A264 (1967), 245-248.

[7] -, Représentation des jets non holonomes par des morphismes vectoriels doubles soudés, ibid. 278 (1974), 1523-1526.

[8] J. V i r s i k, On the holonomity of higher order connections, Cahiers Topologie Géom. Différentielle 12 (1971), 197-212.

Presented to the Semester

Differential Geometry

(September 17-December 15, 1979) 